\title{
Synchronization and Stabilization of Chaotic Dynamics in a Quasi-1D Bose-Einstein Condensate
}

\author{
B. A. Idowu ${ }^{1}$ and U. E. Vincent ${ }^{2,3}$ \\ ${ }^{1}$ Department of Physics, Lagos State University, Ojo, Nigeria \\ ${ }^{2}$ Department of Physical Sciences, Redeemers University, Km 46, Lagos-Ibadan Expressway, Redemption City, Mowe, Nigeria \\ ${ }^{3}$ Department of Physics, Lancaster University, Lancaster LA1 4YB, UK
}

Correspondence should be addressed to B. A. Idowu; babaidowu@yahoo.com and U. E. Vincent; ue_vincent@yahoo.com

Received 10 August 2013; Revised 18 November 2013; Accepted 18 November 2013

Academic Editor: René Yamapi

Copyright (C) 2013 B. A. Idowu and U. E. Vincent. This is an open access article distributed under the Creative Commons Attribution License, which permits unrestricted use, distribution, and reproduction in any medium, provided the original work is properly cited.

\begin{abstract}
A nonlinear control is proposed for the exponential stabilization and synchronization of chaotic behaviour in a model of BoseEinstein condensate (BEC). The active control technique is designed based on Lyapunov stability theory and Routh-Hurwitz criteria. The control design approach in both cases guarantees the stability of the controlled states. Whereas the synchronization of two identical BEC in their chaotic states can be realized using the scheme; a suitable controller is also capable of driving the otherwise chaotic oscillation to a stable state which could be expected in practice. The effectiveness of this technique is theoretically and numerically demonstrated.
\end{abstract}

\section{Introduction}

Chaotic behaviour is a well-known phenomenon in physics, engineering, biology, and many other scientific disciplines. Recently, it has received much attention $[1,2]$, following several efforts to analyse and understand the complexity of nonlinear dynamical systems as well as the studies of feedback control systems design.

The control and synchronization of chaotic systems are challenging problems, since a chaotic system is extremely sensitive to small perturbations. Notwithstanding, the possibility of control and synchronization of chaotic systems under certain conditions has been established [1-8]. Due to the connection between control and synchronization, recent studies cast the problem of synchronization in the framework of control theory. In general, the control problem is aimed at stabilizing a chaotic attractor to either a periodic orbit or an equilibrium point, while for synchronization, two dynamical systems cooperate with each other such that the dynamics of the difference varnishes in the course of time. Many potential applications of these phenomena have come true and remarkable experiments have been done in secure communication, laser, and biological systems and other areas $[1,2]$.

Progress in the research activities on chaos synchronization and stabilization has given birth to various methods such as OGY method [3], PC method [7], active control method [9-13], adaptive control method [14-18], impulsive synchronization technique $[19,20]$, auxiliary system approach [21], backstepping method [22, 23], hybrid control [24], and sliding mode.

In this light, various linear and nonlinear techniques have been proposed for achieving control and synchronization of a wide variety of physical systems. Examples of these abounds. For instance, in ratchet systems where directed transports play prominent role, synchronization has been proposed as a mechanism for controlling directed transports [10, 11, 22, 25]; in lasers, amplified laser output intensity was reported [26] and in long Josephson junctions, the possibility of controlling rapid flux quantum device and phase-locking phenomenon among others have been demonstrated [14, 26-28].

In this paper, first, we modified the active control technique presented by Lei et al. [12] and extended to nonidentical systems $[13,29]$ such that the designed controller inputs based 
on Lyapunov stability theory and Routh-Hurwitz criteria will be used to achieve both the stabilization and synchronization in the Bose-Einstein condensates (BEC). Secondly, the method is applied such that a single control input is sufficient to achieve the stability of the controlled states. Our approach is in contrast to the usual active control technique in which the number of controllers is numerically equal to the dimension of the system. Thus, reducing considerably the controller complexity which is an important factor for practical applications $[23,30]$.

The Bose-Einstein condensates which we study here has been observed in trapped gases of rubidium, sodium, and lithium $[31,32]$. BEC has attracted significant attention due to its potential applications. The creation of BEC has provided a platform for investigating many important phenomena in atomic physics, quantum optics, quantum computation, and so forth. The existence of BEC chaos has been proved and the chaotic properties have also been extensively researched in many previous works (see $[33,34]$ and references therein). Naturally, chaos, which plays a role in the regularity of the system, will cause instability of the condensate wave function. Despite the great interest in the investigation of $\mathrm{BEC}$ chaos, only few recent studies have been devoted to the investigation of chaos control in a BEC. For instance Chong et al. [34] employed the Ott-Grebogi-Yorke (OGY) scheme [3] to examine chaos control in a weakly coupled array of BEC. Similarly, a linear feedback obtained by adjusting the intensity of cold atoms with atomic mirrors was employed to drive the BEC system to regular state [35]. In [36], a parametric modulation of the laser intensity was employed to regulate the BEC dynamics and in particular achieve anticontrol of chaos, in which case periodic states are converted into chaotic states. It is well known that the periodic lattice systems in BEC contain many fantastic properties. For example, quantum computation with BEC atoms in a Mott insulating state is an interesting advancement in applications of the BEC. On the other hand, chaos is associated with quantum entanglement and quantum error correcting which are both the fundamental subjects in quantum computations. Thus, it is valuable to apply or control the chaos in the system.

Recently, vibrational state inversion of a Bose Einstein condensate with optimal control and state tomography was considered in [37], where they presented theoretical and experimental results on high-fidelity transfer of a trapped Bose-Einstein condensate into its first vibrationally excited eigenstate. The excitation is driven by mechanical motion of the trap along a trajectory obtained from optimal control theory. Excellent agreement between theory and experiment is found over a large range of parameters. Similarly, in [38], local exact controllability of a 1D Bose-Einstein condensate in a time-varying infinite square well (box) potential was considered. This is a nonlinear control system in which the state is the wave function of the Bose-Einstein condensate and the control is the length of the box. Here they proved that local exact controllability around the ground state (associated with a fixed length of the box) holds generically with respect to the chemical potential. Vortex synchronization in BoseEinstein condensates vide a time-dependent Gross-Pitaevskii equation approach was considered in [39]. In the work, they consider vortex lattices in rotating Bose-Einstein condensates composed of two species of bosons having different masses. They solved the full two-component Gross-Pitaevskii equations numerically to verify the stability of the putative locked state that is found to exist within a disc centered on the axis of rotation and that depends on the mass ratio of the two bosons.

In this paper, we propose a nonlinear control scheme to realize both chaotic synchronization and exponential stabilization of BEC. In the next section, we formulate the control theories and in Section 3 give a brief description of the system under consideration. Sections 4 and 5 are concerned with the applications of the control techniques for synchronization and stabilization of BEC, respectively. We conclude the paper in Section 6.

\section{Theory}

2.1. Synchronization Principle. Let us consider a driver chaotic system having the same form as in our previous paper [13] and described by

$$
\dot{x}=\mathbf{A} x+f(x),
$$

and a responding system of different form given by

$$
\dot{y}=\mathbf{B} y+f(y)+u(t),
$$

where in (1), $x(t) \in \mathbf{R}^{n}$ is an $n$-dimensional state vector of the system, $\mathbf{A} \in \mathbf{R}^{n}$ is a time-periodic matrix for the system parameter, and $f: \mathbf{R}^{n} \rightarrow \mathbf{R}^{n}$ is the nonlinear part of the system. Similarly in (2), $y(t) \in \mathbf{R}^{n}$ denotes the responding state vector, $\mathbf{B} \in \mathbf{R}^{n}$ is a time-periodic matrix for the response system parameter, and $u(t) \in \mathbf{R}^{n}$ is a controller which is to be designed. If $\mathbf{A}=\mathbf{B}$, then systems (1) and (2) are identical, and the problem reduces to that of [12]; otherwise they are nonidentical.

The dynamic equation of synchronization error, $e=y-x$ can be expressed as

$$
\dot{e}=\mathrm{C} e+f(y)-f(x)+u(t),
$$

where $\mathbf{C}=\mathbf{B}-\mathbf{A}$. Thus, the objective of synchronization is to make $\lim _{t \rightarrow+\infty}\|e(t)\|=0$.

Our goal is to design a suitable control input $u(t)$ to make the dynamical system (3) asymptotically stable at the origin. The active control function $u(t)$ can be redefined as

$$
u(t)=-\mathrm{D} e-f(y)+f(x),
$$

where $\mathbf{D} \in \mathbf{R}^{n}$ is a constant feedback gain matrix.

Then, the error dynamical system (3) can be rewritten as

$$
\dot{e}=\mathbf{M} e,
$$

where $\mathbf{M}=\mathbf{C}-\mathbf{D}$ and $\mathbf{M} \in \mathbf{R}^{n}$.

Theorem 1. If there exists the feedback matrix D such that the eigenvalues of the matrix $\mathbf{M}$ are negative real or complex with negative real parts, then the error dynamical system (5) is globally asymptotically stable at the origin, thus implying that the two nonidentical systems (1) and (2) are globally asymptotically synchronized. 
Remark 2. The proof of Theorem 1 follows immediately as in [12].

2.2. Stabilization Principle. Consider the controlled chaotic system given by (1) and written as

$$
\dot{x}=\mathbf{A} x+f(x)+u(t),
$$

where $u(t)$ is a control input. Without loss of generality, let $P \subset \mathbf{R}^{n}$ be a chaotic bounded set of (6) which is globally attractive, and suppose that $x^{*}=0$ is an equilibrium point embedded in $P$. Stabilizing the chaotic orbits in (6) to its equilibrium point $x^{*}$ implies that the $\lim _{t \rightarrow+\infty}\|e(t)\|=0$, where $e(t)=x-x^{*}$ or $\lim _{t \rightarrow \infty}\|x(t)\|=0$, since $x^{*}=0$, so that $e(t)=x(t)$. For any $n$-dimensional chaotic system, we can follow a recursive procedure and obtain the error dynamics

$$
\dot{e}=\alpha x+f(x)+u(t)
$$

where $\alpha$ is the matrix of the error dynamics. The control input $u(t)$ can be redefined as

$$
u(t)=-\mathbf{D} x+f(x)
$$

where $\mathbf{D} \in \mathbf{R}^{n}$ is a constant feedback gain matrix. Then, the error dynamical system (7) can be rewritten in the form of (5); that is,

$$
\dot{e}=\mathbf{N} x,
$$

where $N=\alpha-\mathbf{D}$ and $\mathbf{N} \in \mathbf{R}^{n}$.

Theorem 3. If there exists the feedback matrix $\mathbf{D}$ such that the eigenvalues of the matrix $\mathbf{N}$ are negative real or complex with negative real parts, then the error dynamical system (9) is globally asymptotically stable at the origin, and the state variable of (6); that is, $x(t) \rightarrow x^{*}$ as $t \rightarrow \infty$.

\section{System Description}

Here, we consider the BEC system described in $[35,36]$, which is created in a harmonically trapped $3 \mathrm{D}$ potential and then loaded into a moving lattice. In principle, near the center of the magnetic trap, the magnetic potential $P_{m}$ is much weaker and when the BEC is formed in this region, $P_{m}$ can be neglected. The quasi-1D potential which plays the main role in the system is valid in the potential region, where $k \sqrt{x^{2}+\left(y^{2} / 2\right)+\left(z^{2} / 4\right)} \leq 100 \pi, k$ being the laser wave vector which fixes the velocity of the travelling lattice as $V_{L}=\delta / 2 k$. By taking into account the effect of damping caused by the incoherent exchange of normal atoms, the system is simply governed by the following Gross-Pitaevskii (GP) equation:

$$
i h(1-i \lambda) \frac{\partial \psi}{\partial t_{1}}=-\frac{\hbar^{2}}{2 m} \frac{\partial^{2} \psi}{\partial x^{2}}+g_{0}|\psi|^{2} \psi+v_{1} \cos ^{2}(k \xi) \psi,
$$

where $m$ is the atomic mass and $g_{0}=4 \pi \hbar^{2} a / m$ represents the interatomic interaction; $a$ being the s-wave scattering length and $\psi$ is the macroscopic quantum equation. Assuming the travelling wave solution $\psi=\varphi(\xi) e^{\left(\alpha x+\beta t_{1}\right)}$ such that the matter wave is a Bloch-like wave (see $[35,36]$ ).

Inserting $\psi$ into (10) we can turn the partial differential equation (10) into an ordinary differential one as follows (see $[35,36]$ for full details):

$$
\begin{aligned}
\frac{\hbar^{2}}{2 m} \frac{d^{2} \varphi}{d \xi^{2}} & +i\left(\frac{\hbar \alpha}{m}+\hbar v_{L}-i \hbar \gamma v_{L}\right) \frac{d \varphi}{d \xi} \\
& -\left(\hbar \beta+\frac{\hbar^{2} \alpha}{2 m}-i \hbar \beta \gamma\right) \varphi-g_{0}|\varphi|^{2} \varphi=v_{1} \cos ^{2}(k \xi) \varphi,
\end{aligned}
$$

where $\alpha$ and $\beta$ are undetermined real constants and the term proportional to $\gamma$ represents the damping effect.

It is convenient to recast equation (11) in dimensionless variables. Now redefining the variables as $t=k \xi, v=$ $2 m v_{L} / \hbar k, \beta_{1}=\hbar \beta / E_{r}, \alpha_{1}=\alpha / k$, and $I_{0}=v_{1} / E_{r}$, and letting $\varphi=R(t) e^{i \theta(t)}$ and $d \theta / d t=-\beta_{1} / v=-(v / 2)+\alpha_{1}$; then (11) in dimensionless form now reads

$$
\ddot{R}=-\mu \nu \dot{R}+\frac{1}{4} v^{2} R+g R_{1}^{3}+I_{o} \cos ^{2}(t) R,
$$

where the over-dots denote the derivative with respect to the re-scaled time, $t, I_{o}$ is optical intensity, and $v=2 m v_{l} / \hbar k$. The square of the amplitude $R$ is just the particle number density because $|R|=|\varphi|=|\psi|$ and $\theta$ is the phase of $\varphi$.

According to the general theory of the Duffing equation, underlying equation (12), has a monoclinic solution only when the coefficients of the linear $(R)$ and nonlinear $\left(R^{3}\right)$ terms on the left-hand side of (12) have opposite signs. Therefore, in order to study the chaos for the negative $R$ term we must consider the case of attractive atom-atom interactions; that is, $g<0$; (12) is just the parametrically driven Duffing equation with a damping term [36].

Solving (12) numerically using the 4th-order Runge-Kutta algorithms with the following initial conditions: $(R, \dot{R})=$ $(1,0)$ and the parameters: $I_{o}=0.5, \mu=0.05, v=2.03$, and $g=-0.75$, the period- 1 attractor (single well) is found as shown in Figure 1 and the period-1 attractor (double well) is displayed in Figure 2, where $I_{o}=0.7$. When the parameters is $I_{o}=5.5, \mu=0.05, v=2.03$, and $g=-0.75$, the strange chaotic attractor projected in the $(R, \dot{R})$ phase plane as shown in Figure 3 is obtained and its time series is shown in Figure 4. Notice that Figure 3 is similar to the plots in $[35,36]$, showing the chaotic state of the BEC. Figure 4 shows the time series of $y_{1}$ and one can find that the value seems random, but it is different from those noise signals without rules and seems to change following some irregularity. If a different initial condition is selected, the trajectories of system (2) would diverge since the system is deterministic and, thus, unpredictable.

\section{Synchronization of Chaos in BEC}

In this section, the method proposed in Section 2 is applied to synchronize the BEC system described in Section 3. Some global asymptotic conditions are first obtained and then further verified by numerical simulations. The synchronization 


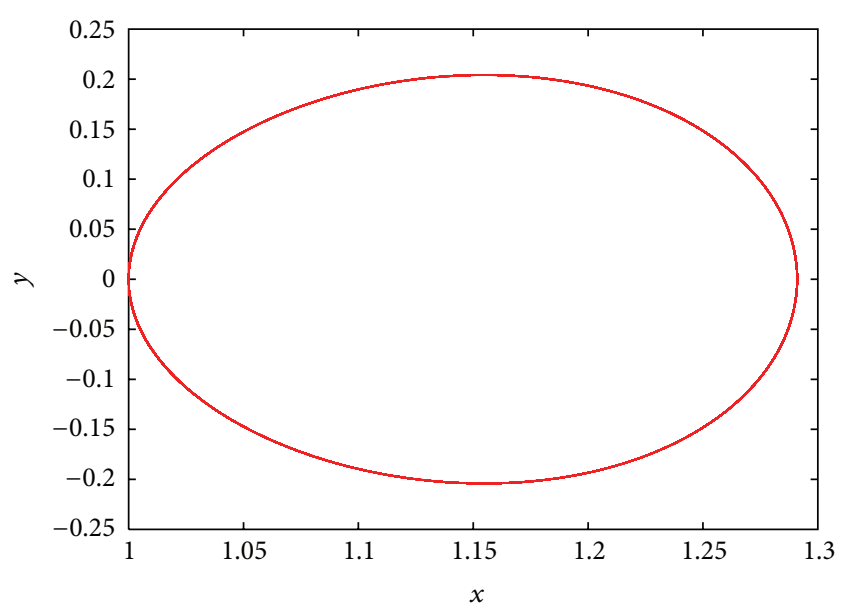

Figure 1: The period-1 attractor (single well) of the BEC with $I_{o}=$ 0.5 .

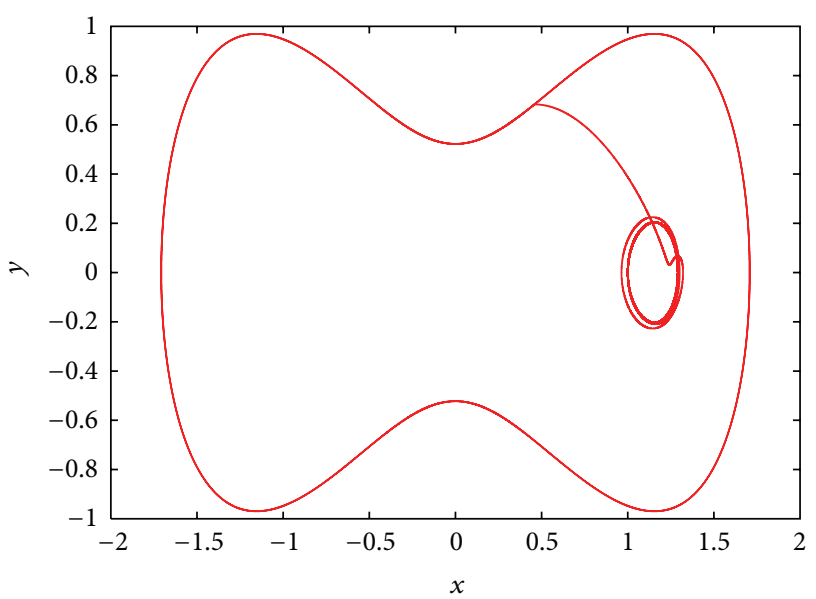

FIGURE 2: The period-1 attractor (double well) of the BEC with $I_{o}=$ 0.7 .

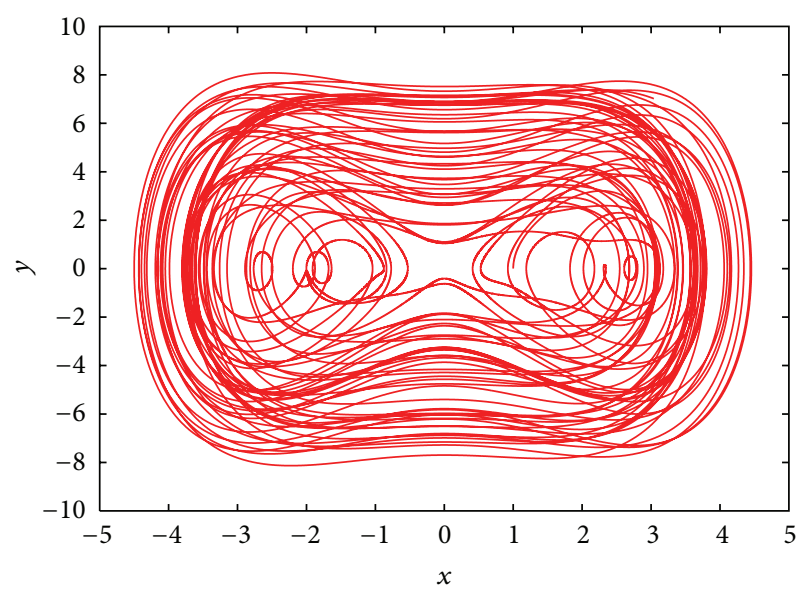

Figure 3: The chaotic attractor of the BEC.

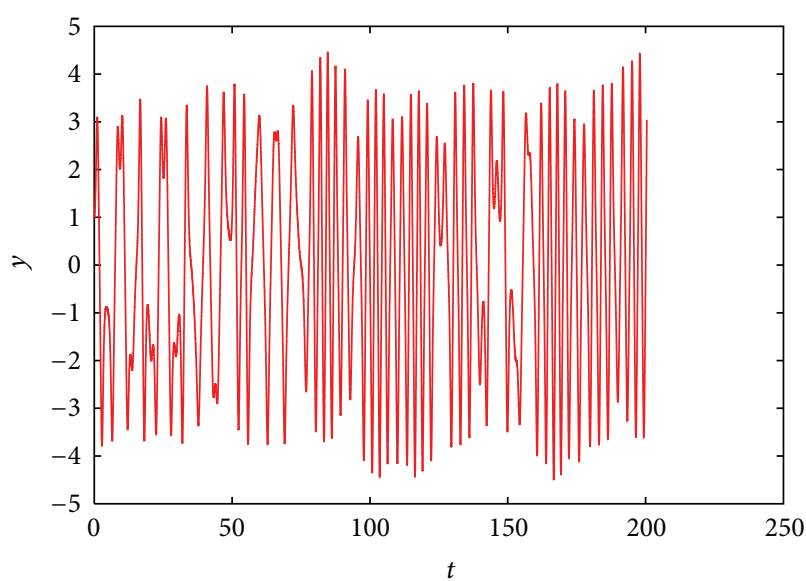

FIGURE 4: The time series of the chaotic attractor of the BEC.

control technique consists of controlling a condensate in the presence of another condensate by means of suitably designed control inputs that links both systems in a drive-response configuration. Let $R=x$ and $\dot{R}=y$; then we expressed the BEC system in drive-response configuration as follows:

$$
\begin{gathered}
\dot{x}_{1}=y_{1}, \\
\dot{y}_{1}=\frac{1}{4} v^{2} x_{1}+g x_{1}^{3}+I_{o} \cos ^{2}(t) x_{1}-\mu v y_{1},
\end{gathered}
$$

for the drive

$$
\begin{gathered}
\dot{x}_{2}=y_{2}+u_{1}(t), \\
\dot{y}_{2}=\frac{1}{4} v^{2} x_{2}+g x_{2}^{3}+I_{o} \cos ^{2}(t) x_{2}-\mu v y_{2}+u_{2}(t),
\end{gathered}
$$

for the response, where $u_{i}(i=1,2)$ are active control inputs to be determined. In order to determine the control functions for system (14), that are required to synchronize it with system (13), we first define the states between the drive-response system as $e_{1}=x_{2}-x_{1}$ and $e_{2}=y_{2}-y_{1}$ and then subtract (13) from (14) to obtain the error dynamics system given by

$$
\begin{gathered}
\dot{e}_{1}=e_{2}+u_{1}(t), \\
\dot{e}_{2}=\frac{1}{4} v^{2} e_{1}+g\left(x_{2}^{3}-x_{1}^{3}\right) I_{o} \cos ^{2}(t) e_{1}-\mu v e_{2}+u_{2}(t) .
\end{gathered}
$$

For convenience, we redefine the active control functions $u_{1}(t)$ and $u_{2}(t)$ as follows:

$$
\begin{gathered}
u_{1}=v_{1}(t), \\
u_{2}=-g\left(x_{2}^{3}-x_{1}^{3}\right)-I_{0} \cos ^{2}(t) e_{1}+v_{2}(t) .
\end{gathered}
$$

Hence, the error system (15) becomes

$$
\begin{gathered}
\dot{e}_{1}=e_{2}+v_{1}(t), \\
\dot{e}_{2}=\frac{1}{4} v^{2} e_{1}-\mu v e_{2}+v_{2}(t) .
\end{gathered}
$$


Equation (17) is the error dynamics, which can be interpreted as a control problem, where the system to be controlled is a linear system with control inputs $v_{1}(t)$ and $v_{2}(t)$ as functions of $e_{1}$ and $e_{2}$. As long as these feedbacks stabilize the system, $\lim _{t \rightarrow \infty}\left\|e_{i}(i=1,2)\right\|=0$. This simply implies that the two BEC systems are synchronized. We can choose the control inputs $v_{1}(t)$ and $v_{2}(t)$ as

$$
\left(\begin{array}{l}
v_{1}(t) \\
v_{2}(t)
\end{array}\right)=\mathbf{D}\left(\begin{array}{l}
e_{1}(t) \\
e_{2}(t)
\end{array}\right),
$$

where $\mathbf{D}=\left(\begin{array}{ll}a & b \\ c & d\end{array}\right)$ is a $2 \times 2$ constant feedback matrix. So that the error system (17) can be rewritten as

$$
\left(\begin{array}{c}
\dot{e}_{1}(t) \\
\dot{e}_{2}(t)
\end{array}\right)=\mathbf{C}\left(\begin{array}{c}
e_{1}(t) \\
e_{2}(t)
\end{array}\right),
$$

where $\mathbf{C}=\left(\begin{array}{cc}a & 1+b \\ c+(1 / 4) v^{2} & d-\mu v\end{array}\right)$ is the coefficient matrix.

According to Lyapunov stability theory and RouthHurwitz criteria, if

$$
\begin{gathered}
a+d-\mu v<0 \\
(1+b)\left(c+\frac{1}{4} v^{2}\right)-a(d-\mu v)<0,
\end{gathered}
$$

then the eigenvalues of the error system (15) must be real or complex with negative real parts and stable synchronized dynamics between the two identical BEC systems is readily achievable.

The 4th-order Runge-Kutta algorithm was used to obtain the numerical solutions of (13) and (14) with a time grid of 0.001. The parameters of the BEC system were fixed as follows: $I_{o}=5.5, \mu=0.05, v=2.03$, and $g=-0.75$, which place the system in the chaotic state. The initial conditions are $\left(x_{1}, y_{1}\right)=(1,0)$ and $\left(x_{2}, y_{2}\right)=(0.3,0.6)$, respectively. For this particular choice of system parameters, it can be readily shown that the matrix $\mathbf{D}$ given by $\mathbf{D}=\left(\begin{array}{cc}0 & 0 \\ -2.03 & -0.8985\end{array}\right)$ satisfy the conditions (18) and the eigenvalues of the error system is negative real or complex with negative real parts, thus leading to the synchronization of systems (13) and (14). Finally the control inputs are

$$
\begin{gathered}
u_{1}=0, \\
u_{2}=k_{0}+k_{1} e_{1}+k_{2} e_{2},
\end{gathered}
$$

where $k_{0}=-g\left(x_{2}^{3}-x_{1}^{3}\right), k_{1}=-2.03$ and $k_{2}=-I_{0} \cos ^{2}(t)-$ 0.8985 .

With the form of (21) satisfying the stability condition (20), we do not need to compute the conditional Lyapunov exponent (CLE) of the systems: more so the negativity of the CLE is only a necessary but not a sufficient condition for stable synchronization [40]. Note that other choices of the feedback gains $\left(k_{1}, k_{2}\right)$ could equally satisfies the conditions (18) and thus lead to synchrony.

The simulation results when the controller (21) is activated are shown in Figure 5. It is obvious that the synchronization error converges to zero asymptotically and the two identical BEC systems indeed achieve chaos synchronization

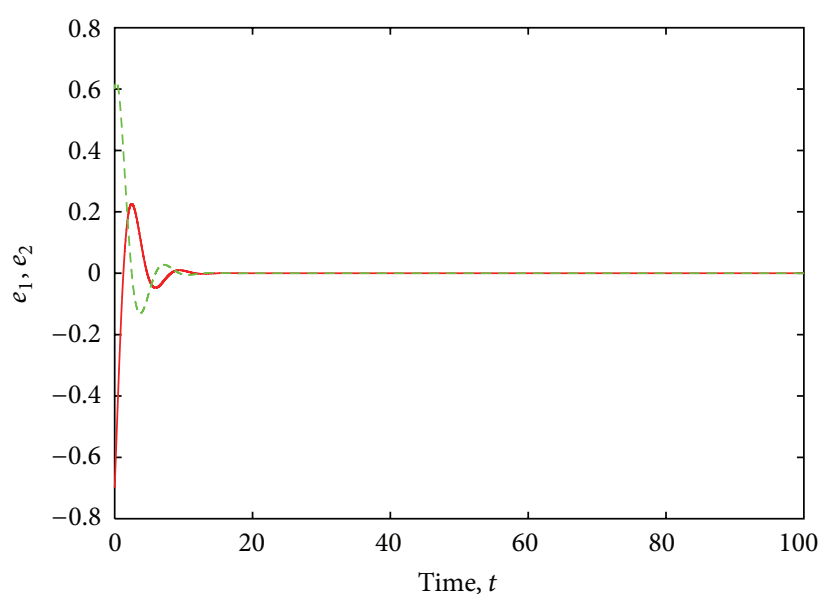

FIGURE 5: The asymptotic convergence of the synchronization error system to zero for identical BEC system.

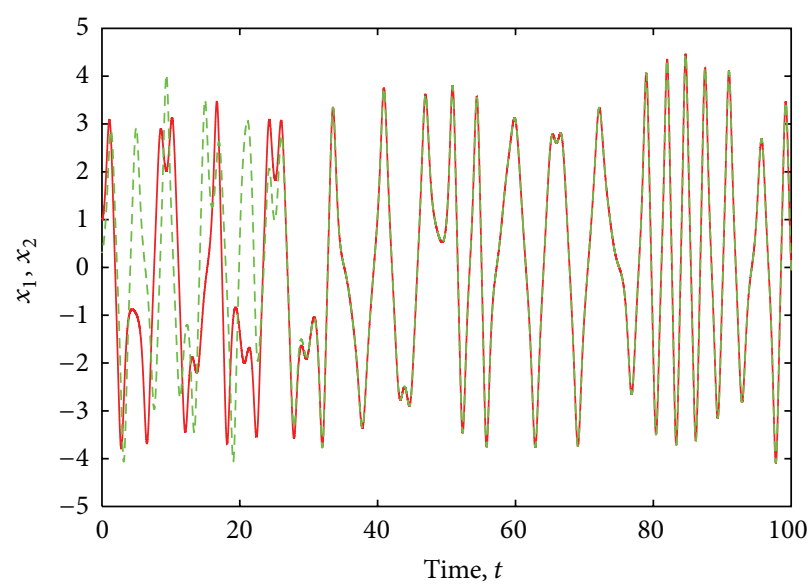

FIGURE 6: The $x_{1}, x_{2}$ variables of the synchronized BEC master and slave system.

as proposed. In Figures 6 and 7, we display the behaviour of the state variables of the master and the slave systems when the control is activated at $t \geq 30$. We see clearly that the slave BEC traces the dynamics of the master BEC. This in particular suggests that the motion of the condensate with a specific dynamics can be driven to follow a desired dynamic state. The idea could be employed to drive a chaotic BEC state to a periodic one and also to achieve the anticontrol (or chaotification) [35].

\section{Stabilization of Chaos in BEC}

Here we propose a recursive active control-to-control the evolution of chaotic state in the BEC system. The goal of the control technique is to drive the BEC system from its current chaotic state $(x, y)$ to a stable regular state $\left(x^{*}, y^{*}\right)$, where a desired dynamic is obtained, since the chaotic property in the spatial distribution of BEC could constitute a disadvantage in many applications. The method proposed by $[35,36]$ relies on the knowledge of the Lyapunov exponent (LE) of the system, 


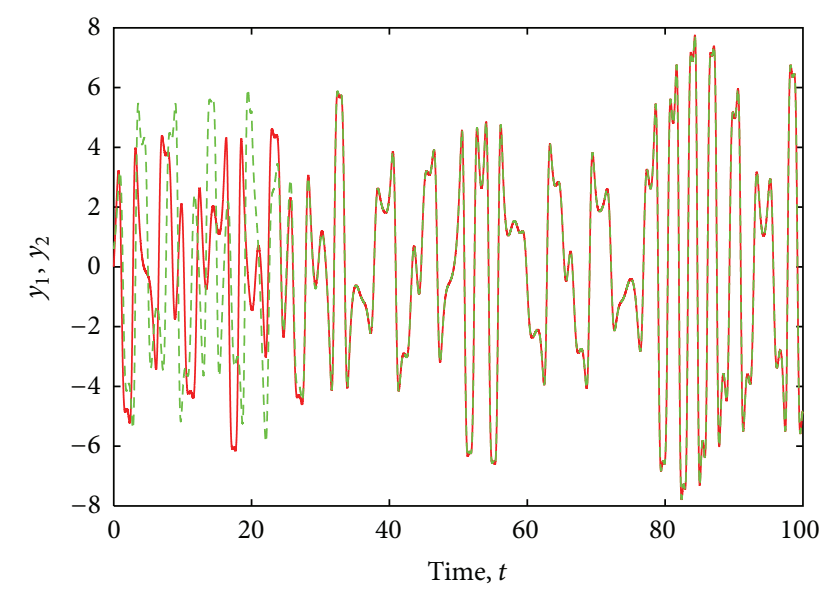

FIGURE 7: The $y_{1}$ and $y_{2}$ variables of the synchronized BEC master and slave system.

which is often time consuming to compute or even difficult in some cases. In our proposed method, the computation of LE is ruled out, while the stability is fully guaranteed using the Lyapunov stability theory and Routh Hurwitz criteria. Now considering the system (22) to be controlled given below

$$
\begin{gathered}
\dot{x}=y+u_{1}(t), \\
\dot{y}=\frac{1}{4} v^{2} x+g x^{3}+I_{o} \cos ^{2}(t) x-\mu v y+u_{2}(t),
\end{gathered}
$$

where $u_{1}$ and $u_{2}$ are control functions to be determined. Starting in a recursive manner, suppose $x^{*}=0$ is an arbitrarily chosen scalar input corresponding to a stable fixed point or an equilibrium point of the system (22) and $y^{*}=c_{1} e_{1}$ is recursively introduced; $c_{1}$ is an arbitrary control parameter. Let the error signal between the current chaotic state and the desired state be given as $e_{1}=x-x^{*}$ and $e_{2}=y-y^{*}$, respectively. Since $(x, y)=(0,0)$ is a stable equilibrium of the system (22), it is convenient to set the arbitrary control parameter $c_{1}=0$ so that $y^{*}=0$, and some simple possible control inputs would drive the system to the state $\left(x^{*}, y^{*}\right)=$ $(0,0)$ that could be obtained. This leads to the following error dynamics system:

$$
\begin{gathered}
\dot{e}_{1}=e_{2}+u_{1}(t) \\
\dot{e}_{2}=\frac{1}{4} v^{2} e_{1}+g e_{1}^{3}+I_{o} \cos ^{2}(t) e_{1}-\mu v e_{2}+u_{2}(t) .
\end{gathered}
$$

Again, we find that the control problem is reduced to that of achieving the asymptotic stabilization of the zero solution of the error dynamics (23). To achieve this, we redefine the active control functions $u_{1}(t)$ and $u_{2}(t)$ as follows:

$$
\begin{gathered}
u_{1}=v_{1}(t), \\
u_{2}=-g e_{1}^{3}-I_{o} \cos ^{2}(t) e_{1}+v_{2}(t) .
\end{gathered}
$$

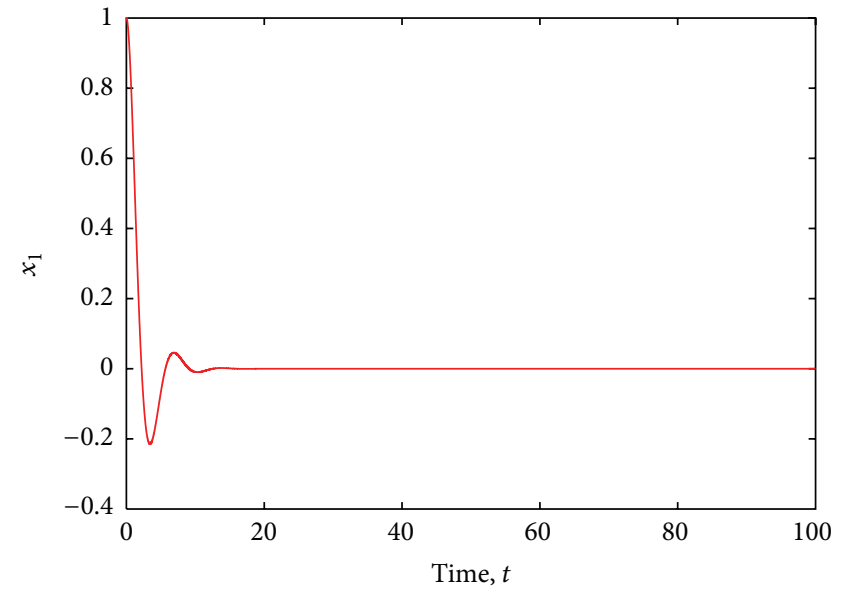

FIgURE 8: The controlled state of the BEC system.

Hence, the error system (23) becomes

$$
\begin{gathered}
\dot{e}_{1}=e_{2}+v_{1}(t), \\
\dot{e}_{2}=\frac{1}{4} v^{2} e_{1}-\mu v e_{2}+v_{2}(t) .
\end{gathered}
$$

The error dynamics of (25) now takes the form of (17) derived in the synchronization process of the two BEC systems. As a result we can apply Lyapunov stability theory and Routh-Hurwitz criteria to system (25) to obtain the control functions. Proceeding as in the previous section and using the same system parameters, one can show that the matrix $\mathbf{D}$ given by $\mathbf{D}(t)=\left(\begin{array}{cc}0 & 0 \\ -2.03 & -0.8985\end{array}\right)$ satisfies the stability criteria. Finally we obtain the following control inputs:

$$
\begin{gathered}
u_{1}=0, \\
u_{2}=k_{0}+k_{1} x+k_{2} y,
\end{gathered}
$$

where $k_{0}=-g x^{3}, k_{1}=-2.03$, and $k_{2}=-I_{0} \cos ^{2}(t)-0.8985$. Notice the nonlinear form of the controller (26) which is in contrast to the proposed controller in [36]. This is expected because in practice, due to some physical limitations, the control input usually contains nonlinear term. Significantly, nonlinearity in systems like BEC could lead to serious degradation of the system performance, decrease in speed of response, and possibly may cause chaotic perturbations to original regular behaviour if in the design process of the controller their effects are ignored [41-43]. Numerically, we implement the controller of (26) in (22), using the 4th order Runge-Kutta algorithm. The result is displayed in Figure 8. Clearly, we see that the chaotic dynamic has been driven to the stable equilibrium point, implying that the BEC chaos is globally controlled.

\section{Conclusions}

In this paper, the synchronization between two-coupled Bose-Einstein Condensates in their chaotic states have been investigated based on a technique derived from nonlinear 
control theory. The nonlinear control is obtained using the active control and the controller is chosen such that a single control input is sufficient to guarantee global stability of the synchronized state. The systematic design approach of the control input does not require the calculation of the Lyapunov exponents; hence, it is simple and convenient. Besides the synchronization of two chaotic BEC systems, the method was also used to obtain a nonlinear control that is capable of driving the otherwise chaotic oscillation to a stable equilibrium state which could be expected in applications. The effectiveness and feasibility of this technique are theoretically and numerically demonstrated. We believe that such nonlinear control would find applications in the experimental control of spatial chaos in BEC. It is likely that nonlinear interactions are necessary for the stability of continuously pumped atom lasers as described in [44]. From the physical point of view, the underlying mechanism is based on the Josephson-type coupling and is well known in the solid state physics as well as in the ultracold quantum superfluid atoms.

According to Haine et al. [45] in the case of a BoseEinstein condensate with a large nonlinear interaction, there is already coupling between different modes of oscillations. This means that each mode of feedback can remove energy from more than one mode of oscillation. This method of extracting energy from the higher modes is quite inefficient. Adding a nonlinear control improves the efficiency of the feedback because it directly removes energy from a larger range of modes.

\section{Acknowledgments}

The research work of U. E. Vincent is supported by the Royal Society of London through the Newton International Fellowship Alumni Scheme. The author acknowledges useful discussions with Peter McClintock.

\section{References}

[1] A. S. Pikovsky, M. G. Rosenblum, and J. Kurths, Synchronization-A Unified Approach to Nonlinear Science, Cambridge University Press, Cambridge, UK, 2001.

[2] M. Lakshmanan and K. Murali, Chaos in Nonlinear Oscillators: Controlling and Synchronization, World Scientific, Singapore, 1996.

[3] E. Ott, C. Grebogi, and J. A. Yorke, “Controlling chaos," Physical Review Letters, vol. 64, no. 11, pp. 1196-1199, 1990.

[4] H. Fujisaka and T. Yamada, "Stability theory of synchronized motion in coupled-oscillator systems," Progress of Theoretical Physics, vol. 69, no. 1, pp. 32-47, 1983.

[5] G. Chen and X. Dong, From Chaos to Order: Methodologies, Perspectives and Applications, World Scientific, Singapore, 1998.

[6] J. Lü, J. Lu, and S. Chen, Chaotic Time Series Analysis and Its Applications, Wuhan University Press, Wuhan, China, 2002.

[7] L. M. Pecora and T. L. Carroll, "Synchronization in chaotic systems," Physical Review Letters, vol. 64, no. 8, pp. 821-824, 1990.
[8] L. Kocarev and U. Parlitz, "Generalized synchronization, predictability, and equivalence of unidirectionally coupled dynamical systems," Physical Review Letters, vol. 76, no. 11, pp. 18161819, 1996.

[9] M. C. Ho and Y. C. Hung, "Synchronization of two different chaotic systems using generalized active network," Physics Letters A, vol. 301, pp. 5-6424-428, 2002.

[10] U. E. Vincent and J. A. Laoye, "Synchronization and control of directed transport in chaotic ratchets via active control," Physics Letters A, vol. 363, no. 1-2, pp. 91-95, 2007.

[11] U. E. Vincent and J. A. Laoye, "Synchronization, antisynchronization and current transports in non-identical chaotic ratchets," Physica A, vol. 384, no. 2, pp. 230-240, 2007.

[12] Y. Lei, W. Xu, J. Shen, and T. Fang, "Global synchronization of two parametrically excited systems using active control," Chaos, Solitons and Fractals, vol. 28, no. 2, pp. 428-436, 2006.

[13] B. A. Idowu, U. E. Vincent, and A. N. Njah, "Synchronization of chaos in non-identical parametrically excited systems," Chaos, Solitons and Fractals, vol. 39, no. 5, pp. 2322-2331, 2009.

[14] R. Guo, U. E. Vincent, and B. A. Idowu, "Synchronization of chaos in RCL-shunted Josephson junction using a simple adaptive controller," Physica Scripta, vol. 79, no. 3, Article ID 035801, 2009.

[15] J. Lu, X. Wu, X. Han, and J. Lü, "Adaptive feedback synchronization of a unified chaotic system," Physics Letters A, vol. 329, no. 4-5, pp. 327-333, 2004.

[16] V. Sundarapandian, "Adaptive control and synchronization of hyperchaotic Lu system," International Journal of Computer Science, Engineering and Information Technology, vol. 1, no. 2, pp. 29-40, 2011.

[17] V. Sundarapandian, "Adaptive control and synchronization of hyperchaotic Newton-Leipnik system," International Journal of Advanced Information Technology, vol. 1, no. 3, pp. 22-33, 2011.

[18] J. Lu, J. Cao, and D. W. C. Ho, "Adaptive stabilization and synchronization for chaotic Lur'e systems with time-varying delay," IEEE Transactions on Circuits and Systems I, vol. 55, no. 5, pp. 1347-1356, 2008.

[19] W. He, F. Qian, J. Cao, and Q. Han, "Impulsive synchronization of two nonidentical chaotic systems with time-varying delay," Physics Letters A, vol. 375, no. 3, pp. 498-504, 2011.

[20] J. Cao, D. W. C. Ho, and Y. Yang, "Projective synchronization of a class of delayed chaotic systems via impulsive control," Physics Letters A, vol. 373, no. 35, pp. 3128-3133, 2009.

[21] W. He and J. Cao, "Generalized synchronization of chaotic systems: an auxiliary system approach via matrix measure," Chaos, vol. 19, no. 1, Article ID 013118, 2009.

[22] U. E. Vincent, "Controlling directed transport in inertia ratchets via adaptive backstepping control," Acta Physica Polonica B, vol. 38, pp. 2459-2469, 2007.

[23] X. Tan, J. Zhang, and Y. Yang, "Synchronizing chaotic systems using backstepping design," Chaos, Solitons and Fractals, vol. 16, no. 1, pp. 37-45, 2003.

[24] P. Li and J. Cao, "Stabilisation and synchronisation of chaotic systems via hybrid control," IET Control Theory and Applications, vol. 1, no. 3, pp. 795-801, 2007.

[25] U. E. Vincent, A. Kenfack, D. V. Senthilkumar, D. Mayer, and J. Kurths, "Current reversals and synchronization in coupled ratchets," Physical Review E, vol. 82, no. 4, Article ID 046208, 5 pages, 2010.

[26] M. R. Parvathi, B. M. Krishna, S. Rajesh, M. P. John, and V. M. Nandakumaran, "Synchronization and control of chaos in 
coupled chaotic multimode Nd:YAG lasers," Physics Letters A, vol. 373, no. 1, pp. 96-101, 2008.

[27] S. K. Dana, P. K. Roy, G. C. Sethia, A. Sen, and D. C. Sengupta, "Taming of chaos and synchronisation in RCL-shunted Josephson junctions by external forcing," IEE Proceedings: Circuits, Devices and Systems, vol. 153, no. 5, pp. 453-460, 2006.

[28] Y. L. Feng and K. Shen, "Chaos synchronization in RCL-shunted Josephson junctions via a common chaos driving," European Physical Journal B, vol. 61, no. 1, pp. 105-110, 2008.

[29] A. N. Njah, "Synchronization via active control of identical and non-identical $\phi^{6}$ chaotic oscillators with external excitation," Journal of Sound and Vibration, vol. 327, no. 3-5, pp. 322-332, 2009.

[30] N. J. Corron, S. D. Pethel, and B. A. Hopper, "Controlling chaos with simple limiters," Physical Review Letters, vol. 84, no. 17, pp. 3835-3838, 2000.

[31] M. H. Anderson, J. R. Ensher, M. R. Matthews, C. E. Wieman, and E. A. Cornell, "Observation of Bose-Einstein condensation in a dilute atomic vapor," Science, vol. 269, no. 5221, pp. 198-201, 1995.

[32] C. C. Bradley, C. A. Sackeu, J. J. Tolleu, and R. G. Hulet, "Evidence of Bose-Einstein condensation in an atomic gas with attractive interactions," Physical Review Letters, vol. 75, no. 9, pp. 1687-1690, 1995.

[33] G. S. Chong, W. H. Hai, and Q. T. Xie, "Spatial chaos of trapped Bose-Einstein condensate in one-dimensional weak optical lattice potential," Chaos, vol. 14, no. 2, pp. 217-223, 2004.

[34] G. S. Chong, W. H. Hai, and Q. T. Xie, "Controlling chaos in a weakly coupled array of Bose-Einstein condensates," Physical Review E, vol. 71, no. 1, Article ID 016202, 2005.

[35] Z.X. Wang and K. Shen, "Anti-control of chaos in Bose-Einstein condensates," Central European Journal of Physics, vol. 6, no. 3, pp. 402-406, 2008.

[36] Z. X. Wang, X. H. Zhang, and K. Shen, "Controlling chaotic behavior in a Bose-Einstein condensate with linear feedback," Communications in Theoretical Physics, vol. 50, no. 1, pp. 215219, 2008.

[37] R. Bucker, T. Berrada, S. van Frank et al., "Vibrational state inversion of a Bose-Einstein condensate: optimal control and state tomography," Journal of Physics B, vol. 46, no. 10, Article ID 104012, 2013.

[38] K. Beauchard, H. Lange, and H. Teismann, "Local exact controllability of a $1 \mathrm{D}$ Bose-Einstein condensate in a time-varying box," http://arxiv.org/abs/1303.2713.

[39] R. Barnett, E. Chen, and G. Refael, "Vortex synchronization in Bose-Einstein condensates: a time-dependent Gross-Pitaevskii equation approach," New Journal of Physics, vol. 12, Article ID 043004, 2010.

[40] J. F. Heagy, T. L. Carroll, and L. M. Pecora, "Desynchronization by periodic orbits," Physical Review E, vol. 52, no. 2, pp. R1253R1256, 1995.

[41] T. Gao, Z. Chen, and Z. Yuan, "Control for the synchronization of Chen system via a single nonlinear input," Journal of Control Theory and Applications, vol. 4, no. 3, pp. 297-301, 2006.

[42] J. J. Yan, "Design of robust controllers for uncertain chaotic systems with nonlinear inputs," Chaos, Solitons and Fractals, vol. 19, no. 3, pp. 541-547, 2004.

[43] Y. Hong, J. Huang, and Y. S. Xu, "On an output feedback finitetime stabilization problem," IEEE Transactions on Automatic Control, vol. 46, no. 2, pp. 305-309, 2001.
[44] S. A. Haine and J. J. Hope, "Mode selectivity and stability of continuously pumped atom lasers," Physical Review A, vol. 68, no. 2, Article ID 023607, 7 pages, 2003.

[45] S. A. Haine, A. J. Ferris, J. D. Close, and J. J. Hope, "Control of an atom laser using feedback," Physical Review A, vol. 69, no. 1, Article ID 013605, 6 pages, 2004. 


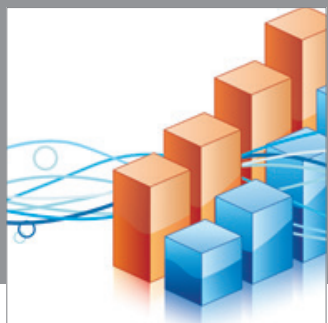

Advances in

Operations Research

mansans

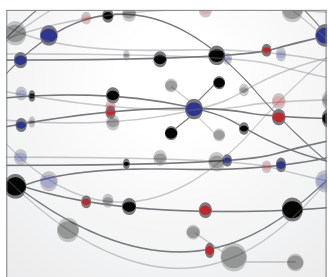

The Scientific World Journal
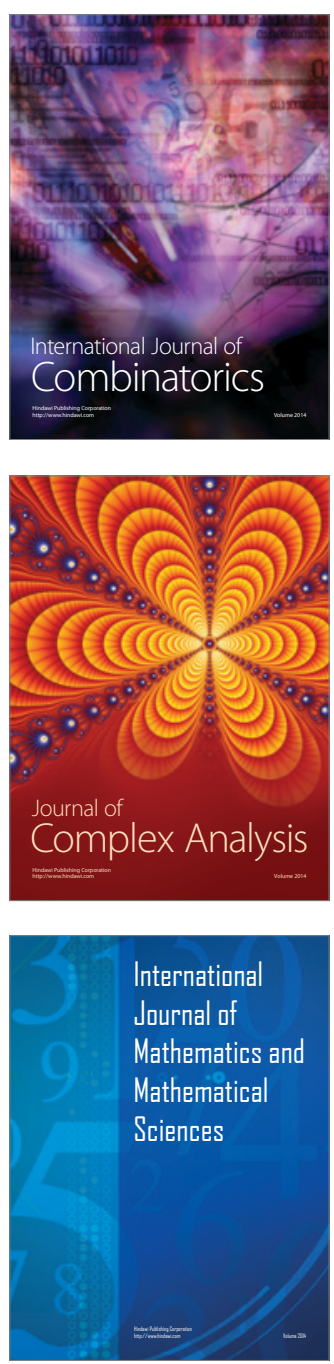
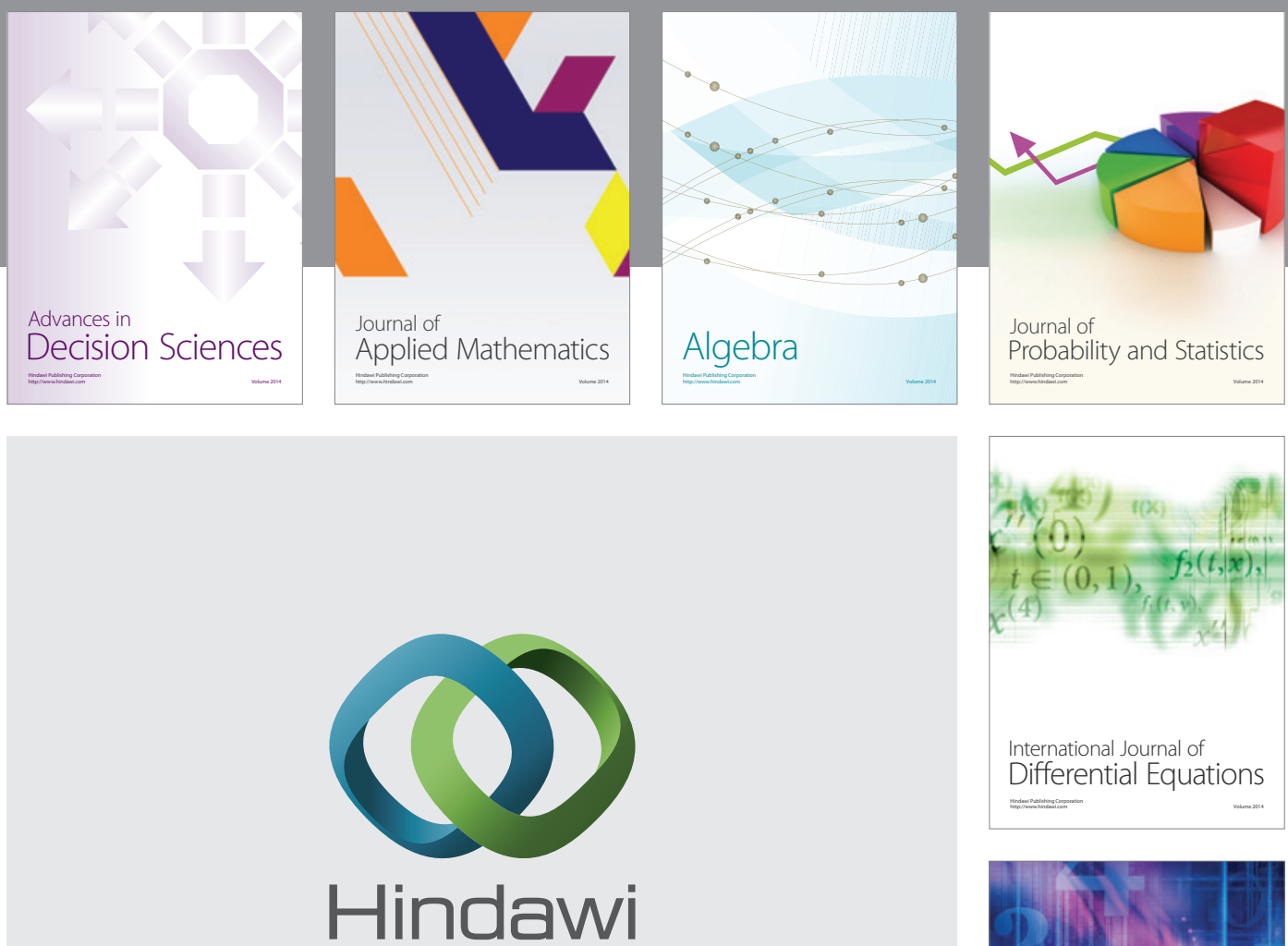

Submit your manuscripts at http://www.hindawi.com
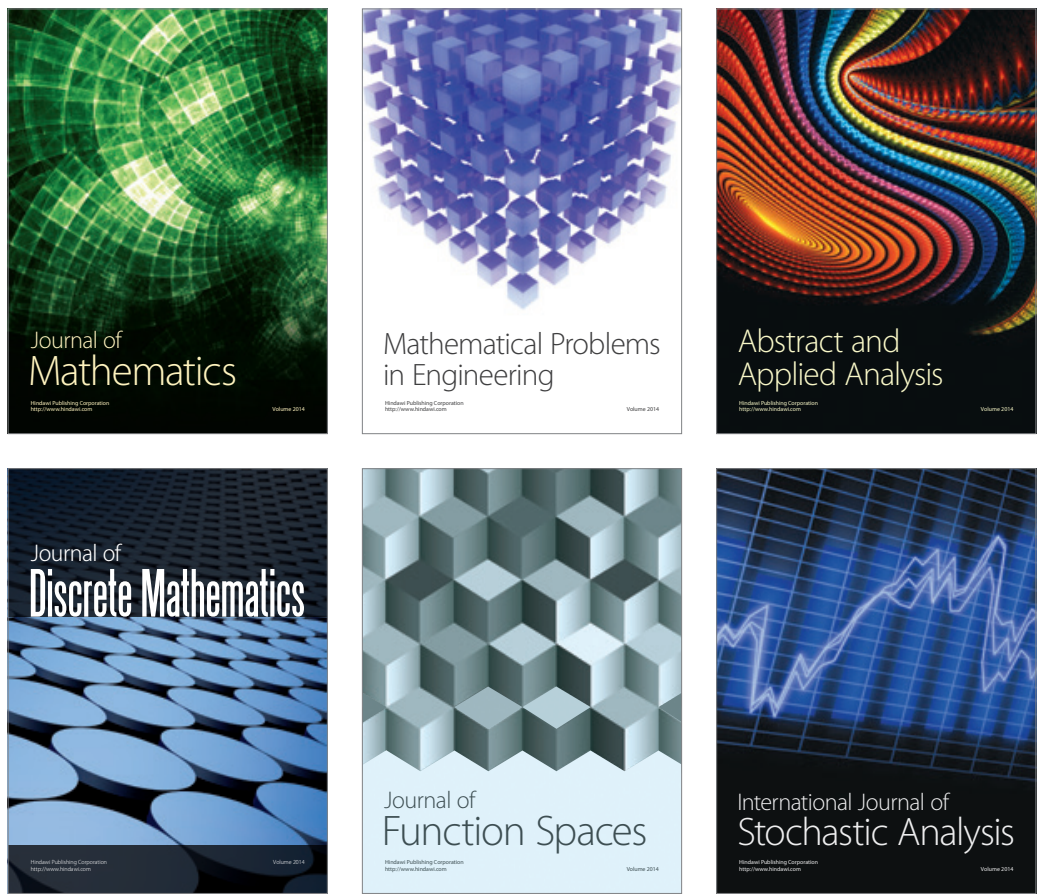

Journal of

Function Spaces

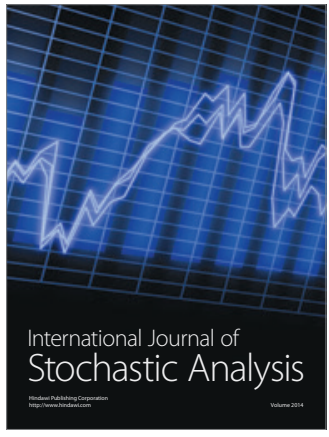

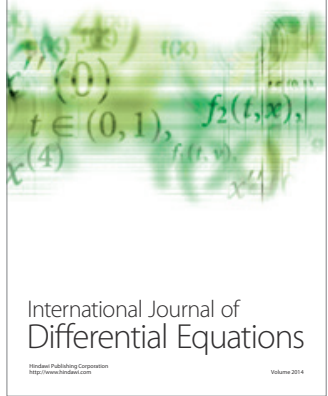
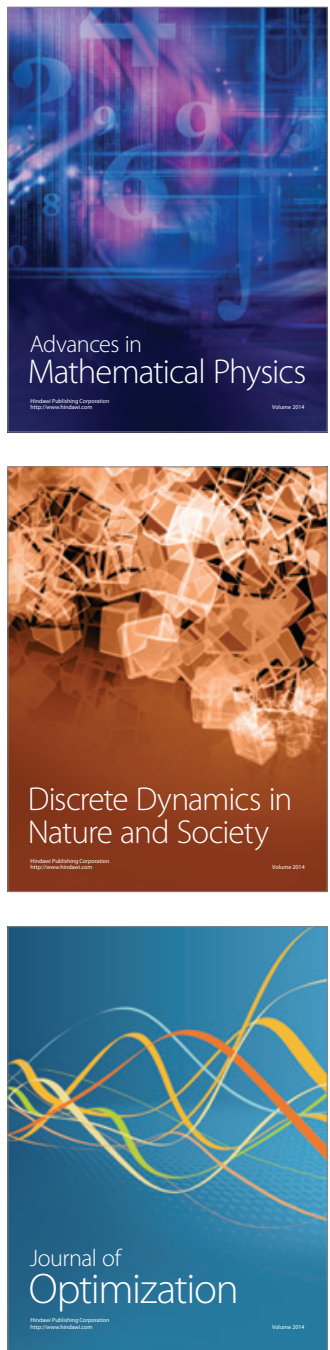